\title{
VIBRATION CHARACTERISTICS OF BEAM STRUCTURE ATTACHED WITH VIBRATION ABSORBER(s) AT ITS VIBRATIONAL NODE AND ANTINODE BY FINITE ELEMENT ANALYSIS
}

\author{
W. S. Ong ${ }^{1, a}$ and M. H. Zainulabidin ${ }^{1, b}$ \\ ${ }^{1}$ Faculty of Mechanical \& Manufacturing Engineering, Universiti Tun Hussein Onn Malaysia, Parit Raja, Batu Pahat, Johor, \\ Malaysia \\ E-mail: wei_syen@hotmail.com
}

\begin{abstract}
In this study, the vibration characteristics of fixed ends beam are analysed after attached with dynamic vibration absorbers at vibrational node and antinode by simulation using ANSYS APDL. This study aim to obtain the best location and optimum number of DVAs placed on the fixed ends beam in order to reduce vibration of beam. The dynamic vibration absorber were attached to the fixed ends beam vibrational node and antinode for a total of three modes of vibration. The $0.84 \mathrm{~m}$ long beam is modelled by ANSYS and divided into 21 elements where each element is $0.04 \mathrm{~m}$. A harmonic force, Fo of $28.84 \mathrm{~N}$ is exerted at node 3 of beam element. Modal analysis and harmonic analysis are carried out in this study to obtain the natural frequency and frequency response of the beam respectively. The vibration characteristics of fixed ends beam without DVA and beam attached with DVAs were compared. The simulation results show reduction of vibration amplitude of the beam especially when the DVA were attached at the vibrational antinode. The DVA amplitude increase when amplitude of beam decreases. From this study, it is proved that DVAs absorb vibration of the beam structure. The best position to attach DVAs is the vibrational antinode based on the modes of vibration. The increment of DVAs number will not affect the percentage reduction of vibration amplitude as long as the DVAs are placed at optimum location.
\end{abstract}

Keywords: node, antinode, vibration absorber, beam, finite element.

\section{INTRODUCTION}

System that oscillates about its equilibrium position or any movements that repeat itself after an interval of time are known as vibration. Vibration may be useful in some case but excessive occurrences of vibrations bring disturbance, discomfort, damage and destruction to both human and system. These excessive vibrations can be reduced by mean of dynamic vibration absorber [1].

In stationary or standing wave, there are certain points that undergo zero amplitude (node) and maximum amplitude (antinode). The nodes and antinodes will remain fixed at its positions all the time with regular spacing. , it is relevant to be able to predict the antinode accurately, points of the maximum displacement. This is because one may want to install vibration absorbers or incorporate additional constrains to the system at this points in order to reduce the response of the system [2].

The tuned vibration absorber act to alter the natural frequencies and mode shapes by attach one or more spring-mass system to the structure. Its mass only needs to be a few percent of primary mass with greater motion in order to obtain same natural frequency as the frequency of excitation. The tuned vibration absorber is compelling when the excitation frequency is almost the same as natural frequency of primary system [3].

The first vibration absorber which consists of a spring-mass system that attached to the main device is proposed by Frahm in 1909. The additional system is function to prevent the main device from vibratie at the frequency of the sinusoidal force [4]. The principle of this DVA is when the primary system has excessive vibration at an operating frequency which approaches its natural frequency, the absorber system need to operate in a natural frequency that same as the primary system in order to reduce the amplitude to zero [5]. According to the study conducted by Zainulabidin and Jaini (2012), the installation of DVAs on the beam successfully absorb the vibration of the beam which the vibration amplitude is reduced in all 4 different conditions based on experiment [6]. The study is then proved by Aslamiah (2016) using finite element analysis (FEA) method with ANSYS APDL software. Dynamic vibration absorbers are effectively reduced the amplitude of vibration for some vibration modes [7]. A finite element modeling of fixed-fixed end plate attached with vibration absorber is carried out by Salleh and Zaman (2016). It is proved that usages of single lightweight dynamic vibration absorber perform better than dual lightweight dynamic vibration absorber in absorb vibration of fixed ends plate [8].

Finite element method (FEM) is a numerical method which it is use to solve differential or integral equation. Finite element method used to simplify most of the complex and insolvable problems. The basic idea of 
FEM is by breaking up the geometry of the body into finite, simple shaped elements [9].

In this paper, a simple vibration absorber system which is spring-mass system is used to absorb the vibration on fixed ends beam. The study is carried out by finite element analysis using ANSYS ADPL. This study is aim to investigate the optimum location at either antinode or node for DVAs placement to achieve best vibration attenuation so that the results can be used as a reference for DVAs placement. Besides that, this study also aims to determine the optimum number of DVAs to prevent wastage.

\section{METHODOLOGY}

The total length, $\mathrm{L}$ of the beam is $0.84 \mathrm{~m}$ and fixed at both of its ends. The beam modulus of elasticity, density and mass moment are $69 \mathrm{GPa}, 2800 \mathrm{kgm}^{-3}$ and $1.67 \times 10^{-11}$ $\mathrm{kgm}^{2}$ respectively. The mass of dynamic vibration absorber is $0.1 \mathrm{~kg}$ while the spring stiffness is adjusted so that the values are equal to the beam 1 st three modes of natural frequency respectively.

There are two categories of testing condition for this study which the first category was to study the effect of tuning on DVA performance at either node or antinode of vibration modes. The DVAs that specifically tuned to natural frequencies of each vibration mode is placed at node or antinode for first three modes of vibration. The second category testing was used to study the effect of multiple DVA placed at either node or antinode of different mode of vibration.

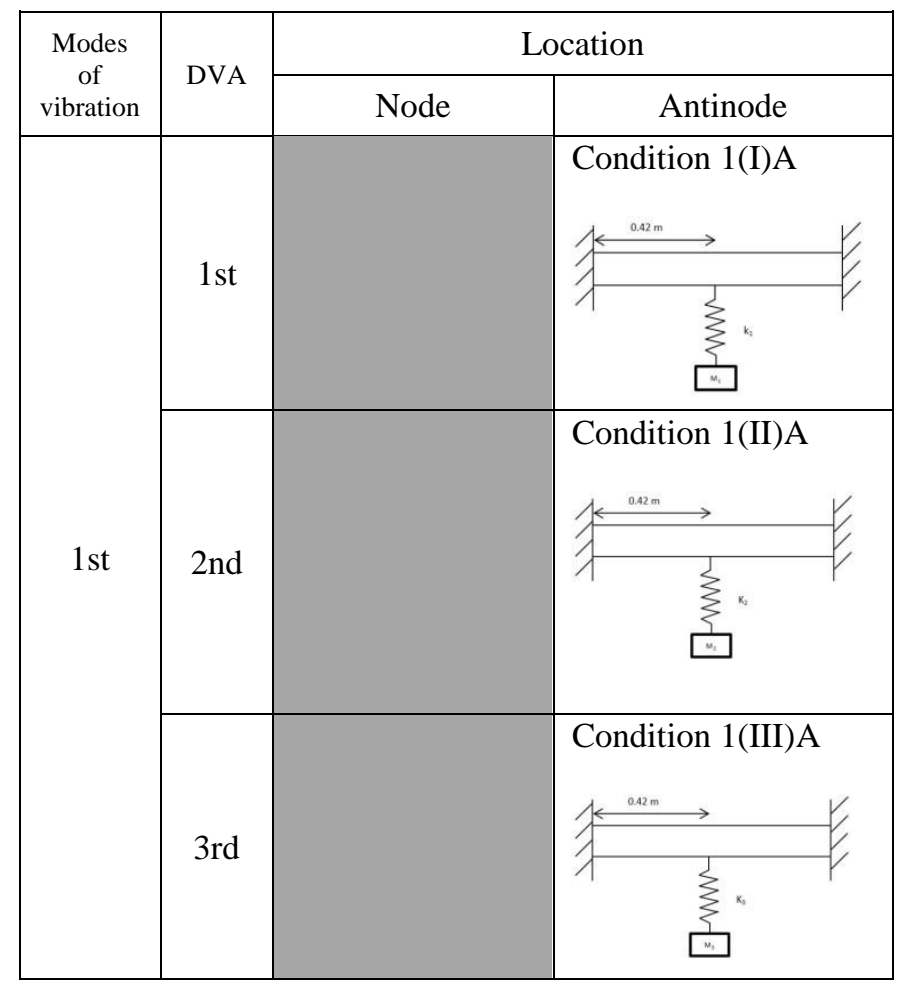

Conditions for first category testing are:

3 DVA was specifically tuned to match the natural frequency for first three modes of vibration and each DVA will be placed on vibrational node and vibrational antinode

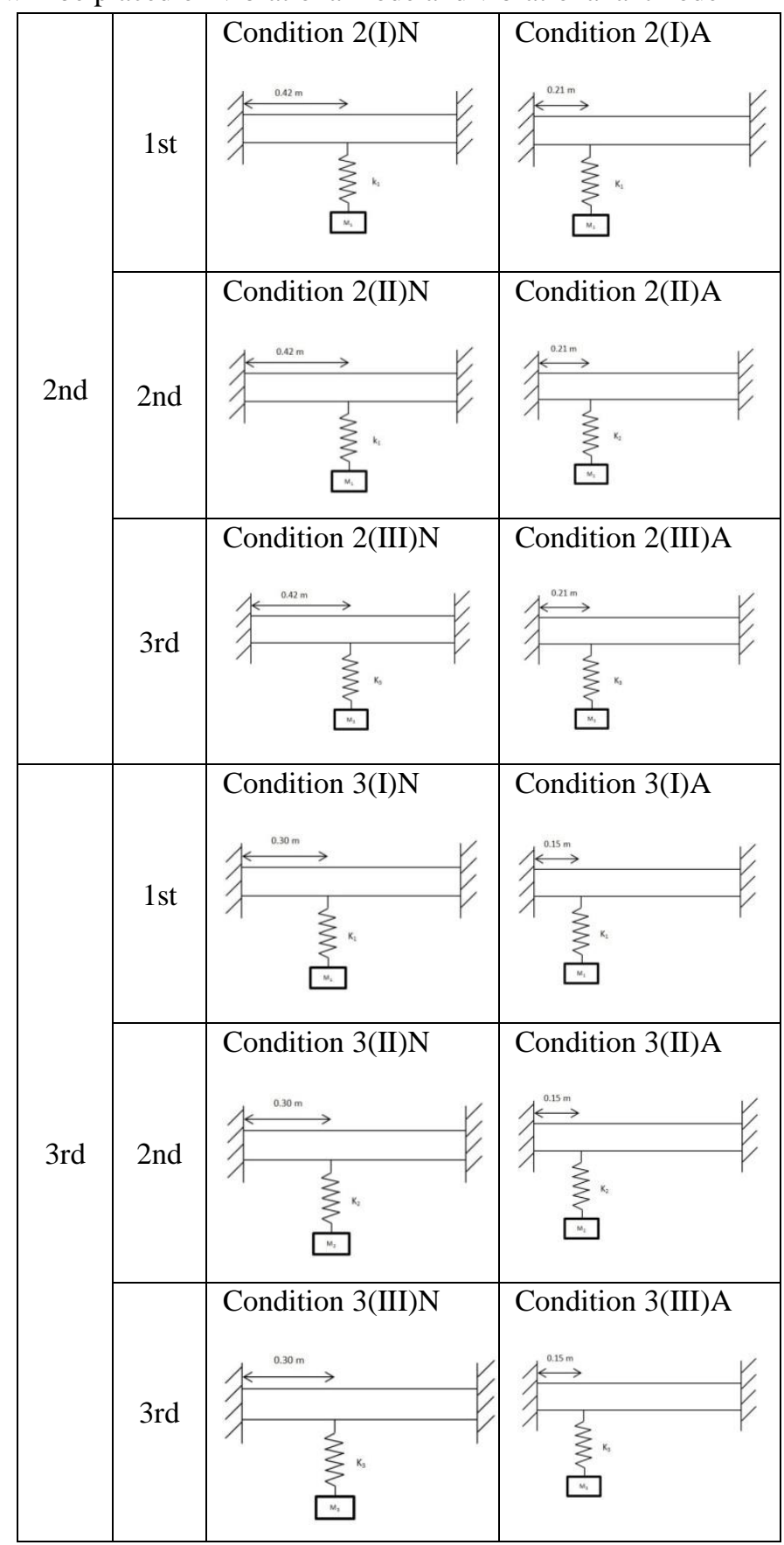

as shown in Table-1. The condition is labeled by number which indicate the modes of vibration, roman number which indicate type of DVA and alphabet ' $N$ ' represent DVA placement at node while alphabet ' $A$ ' indicate DVA placement at antinode.

Table-1: Condition for first category of testing

Conditions for second category testing are:

Condition A: First DVA and second DVA attached at antinode of first vibration mode and second vibration mode respectively 
Condition B: First DVA and third DVA attached at antinode of second vibration mode and third vibration mode respectively

Condition C: Second DVA and third DVA attached at node of second vibration mode and third vibration mode respectively

Condition D: Second DVA and third DVA attached at antinode of second vibration mode and third vibration mode respectively

Condition E First DVA, Second DVA and third DVA attached at antinode of first vibration mode, second vibration mode and third vibration mode respectively

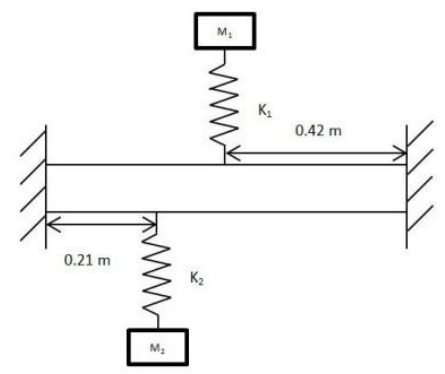

(A)

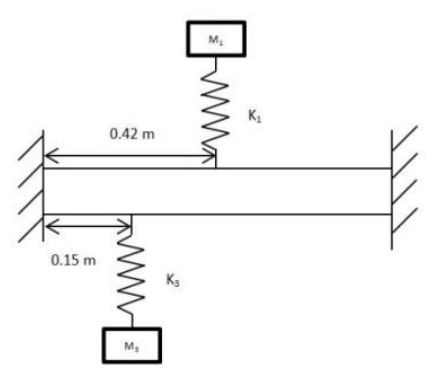

(B)

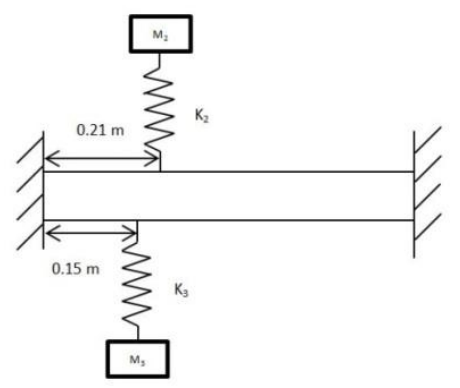

(C)

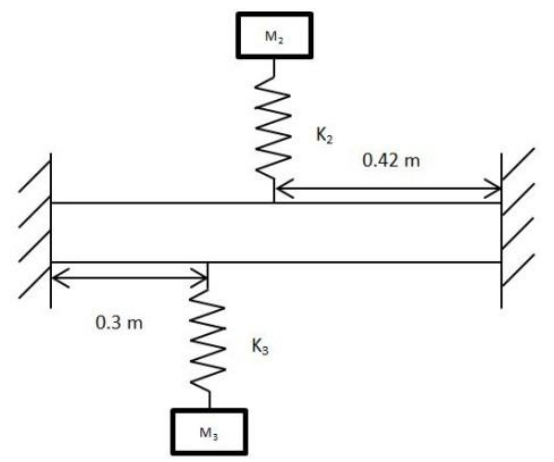

(D)

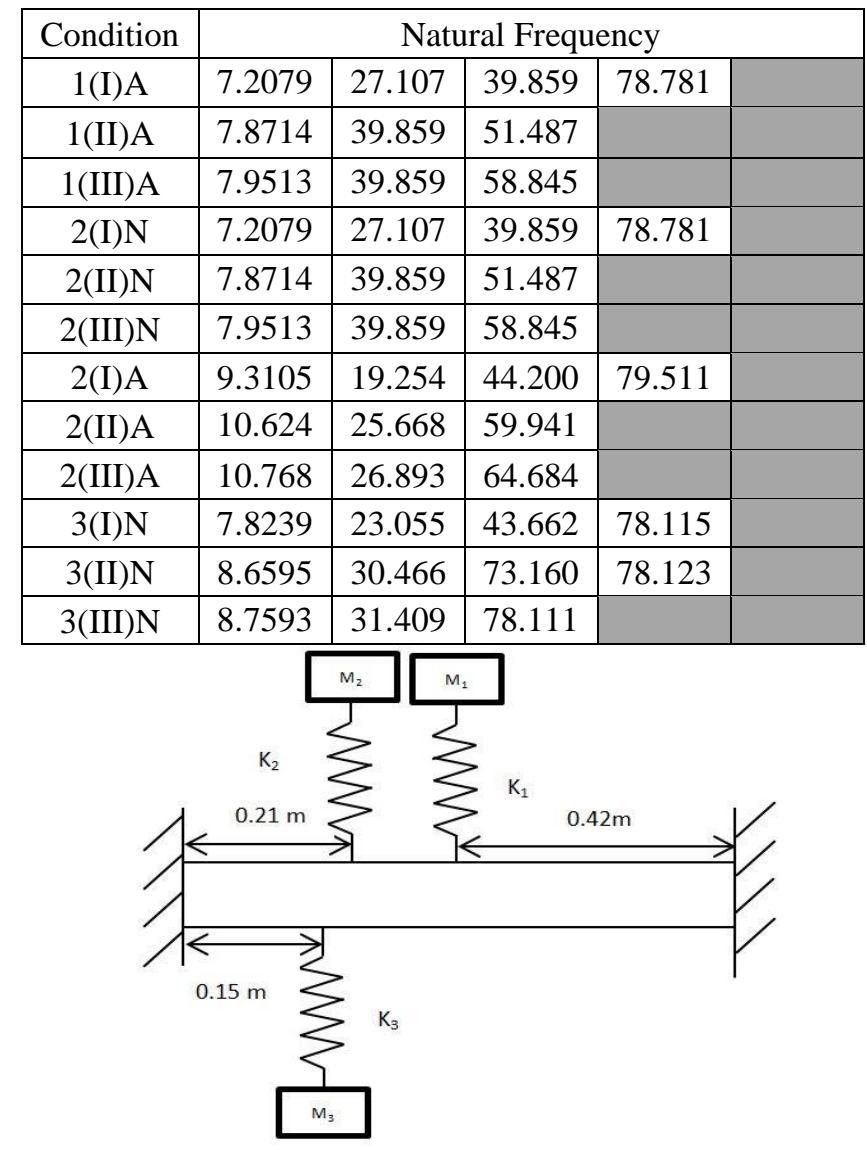

(E)

Figure-1. (A)-(E) Schematic of the conditions set

The beam structure was modelled by BEAM3 element and being divided into 21 elements. The displacement and rotation of beam ends are constrained since it is fixed ends beam. The COMBINI14 element type is used to build the spring element of DVA where the study only considers the longitudinal capability without damping capability. MASS21 is used to combine with spring system using COMBIN14 to create a dynamic vibration absorber system. The auxiliary masses were constrained such that it will only move in vertical direction.

Type of analysis that involved using Mechanical ANSYS Parametric Design Language (APDL) 15.0 was Modal Analysis and Harmonic Analysis. Modal analysis was used to determine the natural frequencies and mode shapes of a structure. Harmonic analysis provides the ability to predict the sustained dynamic behaviour of the structure. The analysis was used to determine the steadystate response of a linear structure to loads that vary harmonically with time. It is to calculate the structure's response at several frequencies which usually will produce a graph of amplitude versus frequency. 


\section{RESULTS \& DISCUSSION}

\section{Modal analysis of fixed ends beam}

Modal analysis is performed using ANSYS APDL to determine the natural frequency of the fixed ends beam. This is because the vibration will occur at its natural frequencies and excitation force that occurs at the same natural frequency will lead to vibration resonance. The value for natural frequency for first, second and third mode of vibration are $14.464 \mathrm{~Hz}, 39.870 \mathrm{~Hz}$ and $78.163 \mathrm{~Hz}$ respectively. The natural frequency of beam with or without absorber masses are determined to obtain the effect of these masses toward beam natural frequencies. Besides that, the natural frequency of beam attached with dynamic vibration absorbers are as showed in Table-2. Since the system will change from single degree of freedom to two degree of freedom after attached with dynamic vibration absorber, the new natural frequency of the system is recorded in range of $0 \mathrm{~Hz}$ to $90 \mathrm{~Hz}$. This is to observe the characteristics of new natural frequencies within the range as first three vibration modes occur within frequency of $14 \mathrm{~Hz}$ to $78 \mathrm{~Hz}$.

Table-2: Natural frequency of the fixed ends beam attached with dynamic vibration absorber

\begin{tabular}{|c|c|c|c|c|c|}
\hline 3(I)A & 10.849 & 17.039 & 41.845 & 78.257 & \\
\hline 3(II)A & 12.559 & 24.341 & 50.726 & & \\
\hline 3(III)A & 12.695 & 26.186 & 54.909 & & \\
\hline A & 6.7673 & 17.294 & 31.036 & 61.441 & \\
\hline B & 7.0125 & 12.750 & 27.914 & 41.903 & 78.828 \\
\hline C & 6.5007 & 24.408 & 47.577 & 88.770 & \\
\hline D & 9.7027 & 23.965 & 42.564 & 59.971 & \\
\hline E & 6.6075 & 15.394 & 30.464 & 42.563 & 61.500 \\
\hline
\end{tabular}

Harmonic analysis of fixed ends beam without absorber Harmonic analysis was carried out to study the frequency response of fixed ends beam. The graph in Figure-2 shows that the frequency response of the fixed ends beam without DVA.

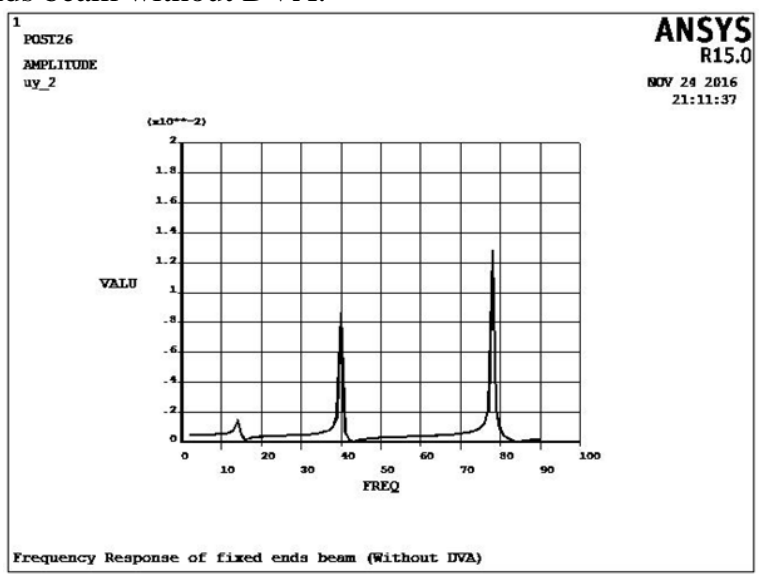

Figure-2: Frequency response of a beam without DVA

The three peaks show the vibration amplitude that occurs in first three modes of vibration at approximate 14 $\mathrm{Hz}, 40 \mathrm{~Hz}$ and $78 \mathrm{~Hz}$. First mode of vibration at $14 \mathrm{~Hz}$ carry an amplitude of $1.45 \times 10^{-3} \mathrm{~m}$, second mode of vibration at $40 \mathrm{~Hz}$ carry an amplitude $8.61 \times 10^{-3} \mathrm{~m}$ of while third mode of vibration carry an amplitude of $1.28 \times 10^{-2} \mathrm{~m}$. The changes of amplitude in these three frequencies are observed and evaluated after attached with DVA in order to calculate the reduction of amplitude for each condition set.

Harmonic analysis of fixed ends beam with absorbers First test: Study effect of tuning on DVA performance Second test: Study effect of multi DVA placement at either node or antinode of the beam.

\section{Effect of each DVA toward first mode of vibration}

Fifteen types of condition are set for this simulation where DVA of frequency $14.464 \mathrm{~Hz}$ namely first DVA, $39.870 \mathrm{~Hz}$ namely second DVA, $78.161 \mathrm{~Hz}$ namely third DVA is placed on the node and antinode of first three modes of beam vibration respectively. Since, the location for antinode of first vibration mode is same as the location for node of second vibration mode which is at middle of the beam, it will contribute to same result. Thus, it will be analyses at once.

\section{Result of the Condition 1(I)A/ Condition 2(I)N}

From the frequency response of DVA show in Figure-3, it shows that the DVA absorb the first vibration mode of beam and created two resonance frequencies. Second mode of vibration didn't show huge changes. Although the First DVA is use to neutralize first mode of vibration, the additional of mass at the middle of the beam which is also the location of first and third mode of the vibration antinode has altered the natural frequency for both first mode and third mode of vibration.

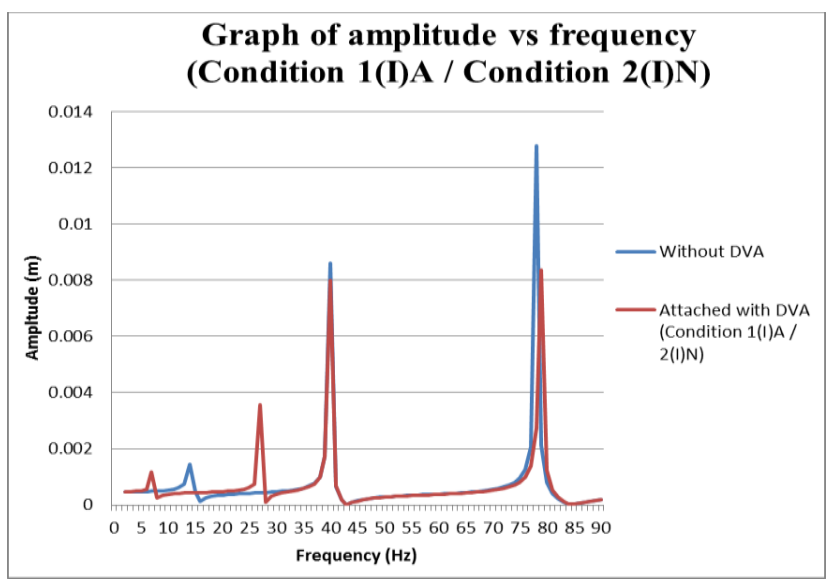

Figure-3: Graph of frequency response comparison between beam without DVA and Condition 1(I)A/ Condition 2(I)N

\section{Result of the Condition 1(II)A/ Condition 2(II)N}

Comparison between fixed ends beam attached with DVA and fixed ends beam without DVA is can be seen through line graph in Figure-4. It shows that the DVA successful absorb first and third mode of vibration. The vibration reduction occurs is due to effect of mass in DVA. This is because the DVA placed at the middle of the beam 
which is also the antinode of first and third mode of vibration alters the natural frequency of first and third mode. On the other side, the location denotes as the node for second vibration mode, hence, the reduction in second mode is consider zero.

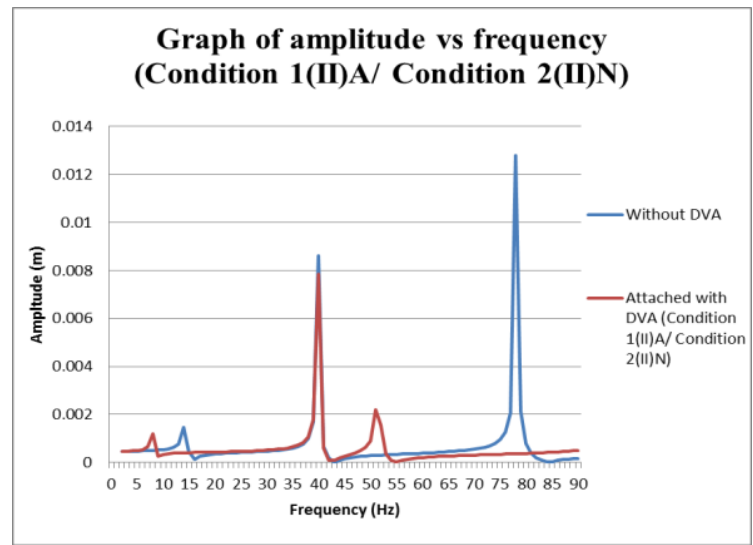

Figure-4: Graph of frequency response comparison between beam without DVA and Condition 1(II)A/ Condition 2(II)N

\section{Result of the Condition 1(III)A/ Condition 2(III)N}

From the graph in Figure-5, it shows that it have similar trend as 'Condition 1(II)A/ Condition 2(II)N' where only the amplitude for first and third mode drop drastically. The mass of the DVA had affected the natural frequency for first and third mode which eventually contributes to vibration absorption to particular mode of vibration.

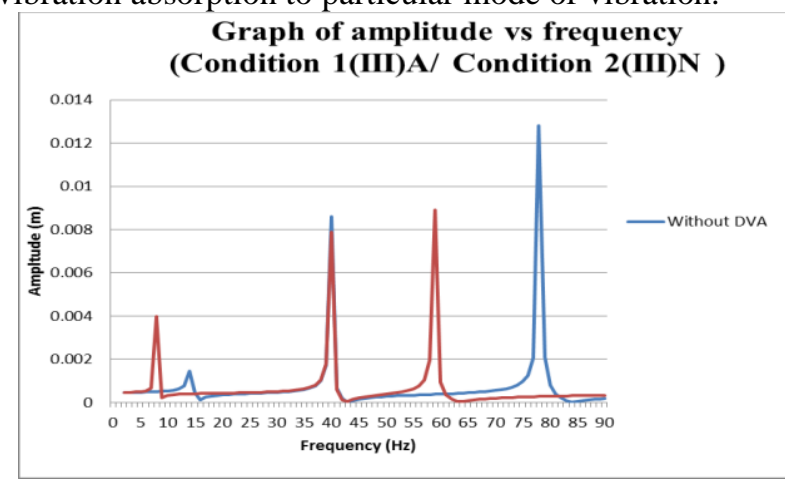

Figure-5: Graph of frequency response comparison between beam without DVA and Condition 1(III)A/ Condition 2(III)N

\section{Result of the Condition 2(I)A}

From the graph in Figure-6, it shows that all the amplitude of vibrations reduced. The new natural frequencies of the system shifted near to initial system. The mass of DVA plays an important role in changing the natural frequencies of the system.

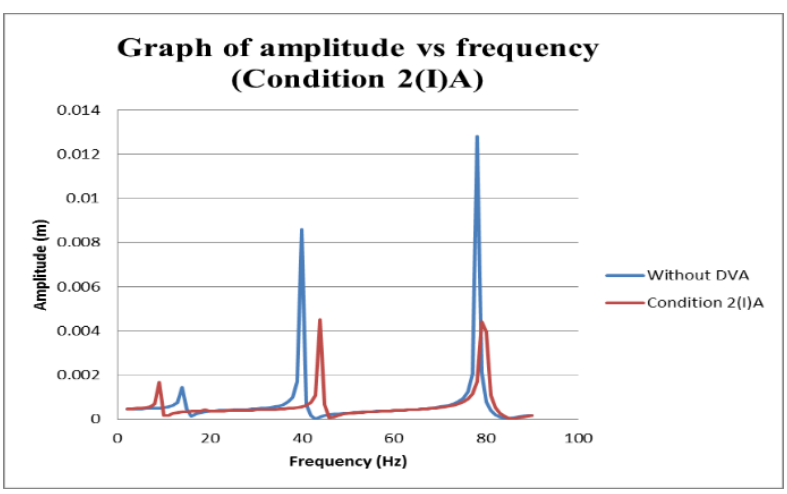

Figure-6: Graph of frequency response comparison between beam without DVA and Condition 2(I)A

\section{Result of the Condition 2(II)A}

A clear comparison of amplitude reduction can be obtained from Figure-7. All three modes of vibration show a drastic reduction in amplitude. This is due to the location of DVA that placed exact at antinode of second vibration mode and near to antinode of first and third vibration. The mass of DVA contribute to the reduction of amplitude due to shifting of natural frequency.

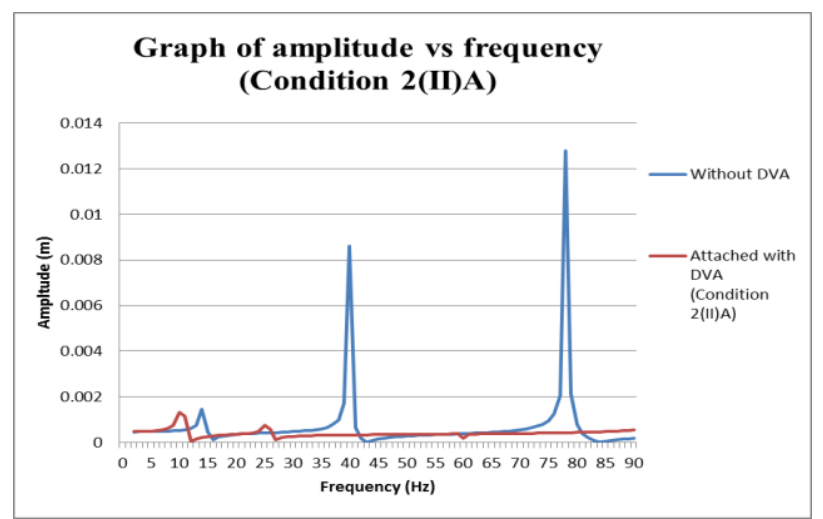

Figure-7: Graph of frequency response comparison between beam without DVA and Condition 2(II)A

\section{Result of the Condition 2(III)A}

From the graph in Figure-8, It shows that DVA that placed at the antinode of second vibration manage to absorb all the three modes of vibration. Mass and location of DVA contribute to this result.

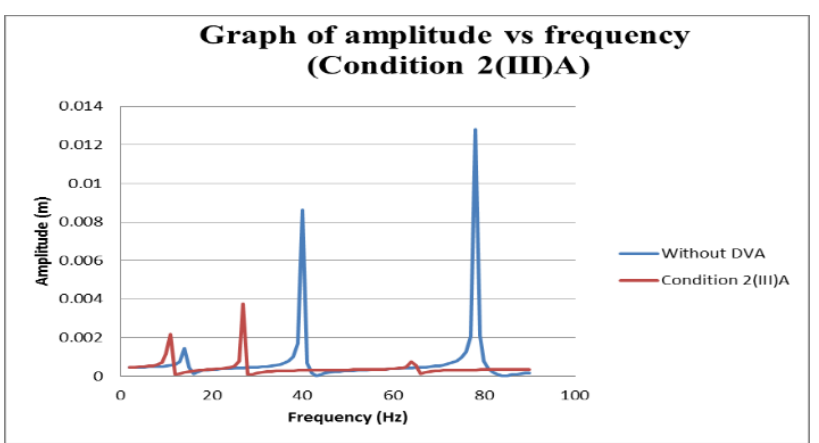

Figure-8: Graph of frequency response comparison between beam without DVA and Condition 2(III)A 


\section{Result of the Condition 3(I)N}

From the graph in Figure-9, first and second modes of vibration reduced drastically. However, the amplitude of third mode of vibration increased up to $38 \%$. This is due to DVA that placed at the node of third vibration mode.

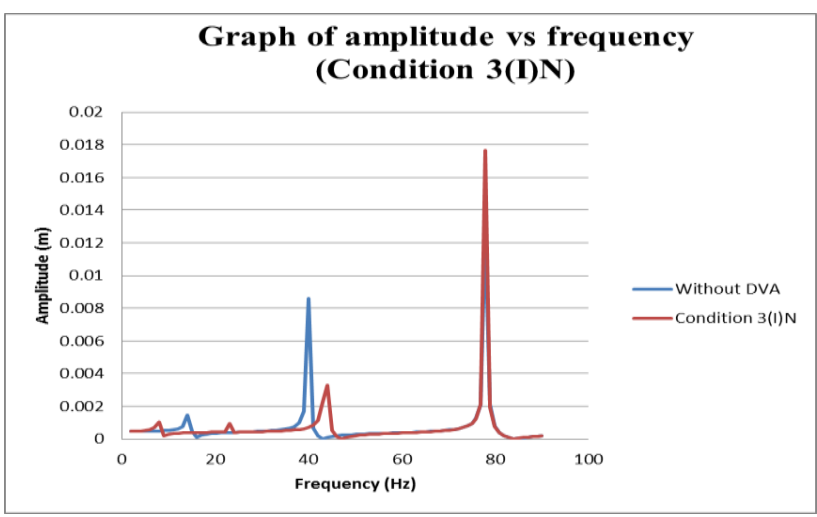

Figure-9: Graph of frequency response comparison between beam without DVA and Condition 3(I)N

\section{Result of the Condition 3(II)N}

First and second mode vibration manage to be reduced while amplitude of third mode vibration increased to $1.74 \times 10^{-2} \mathrm{~m}$. The increment in amplitude indicate that the beam vibrate more vigorously at third mode frequency after attaching with DVA.

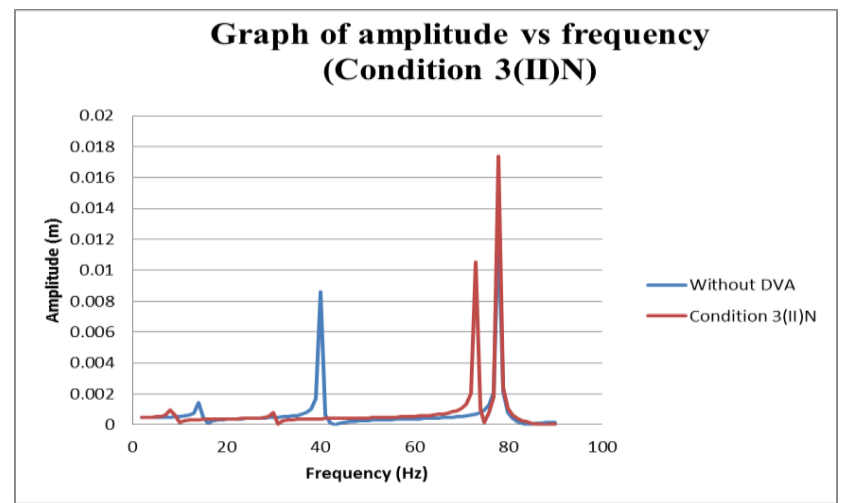

Figure-10: Graph of frequency response comparison between beam without DVA and Condition 3(II)N

\section{Result of the Condition 3(III)N}

From Figure-11, the DVA manage to absorb first and second mode of vibration due to its mass and location. However, the amplitude of third vibration mode increases because of inertia effect of the mass.
Graph of amplitude vs frequency (Condition 3(III)N)

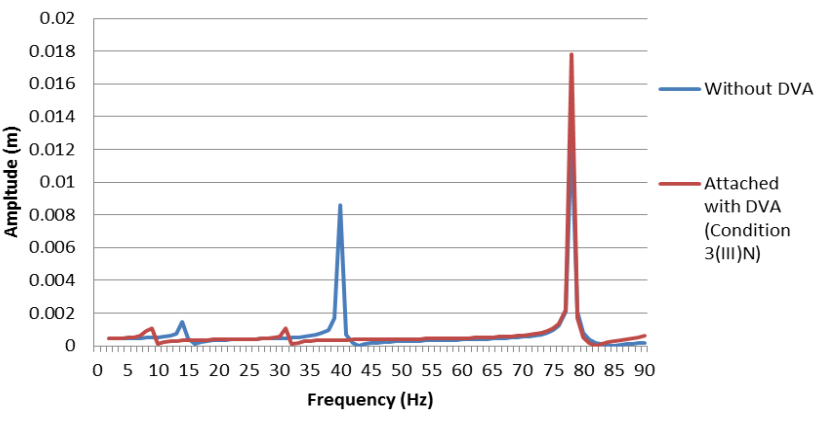

Figure-11: Graph of frequency response comparison between beam without DVA and Condition 3(III)N

\section{Result of the Condition 3(I)A}

Placement of first DVA manages to decrease all the three modes of vibration. However, third mode of vibration undergoes reduction of $37.42 \%$ only. This is mainly due to huge difference between third mode natural frequency and DVA tuning frequency.

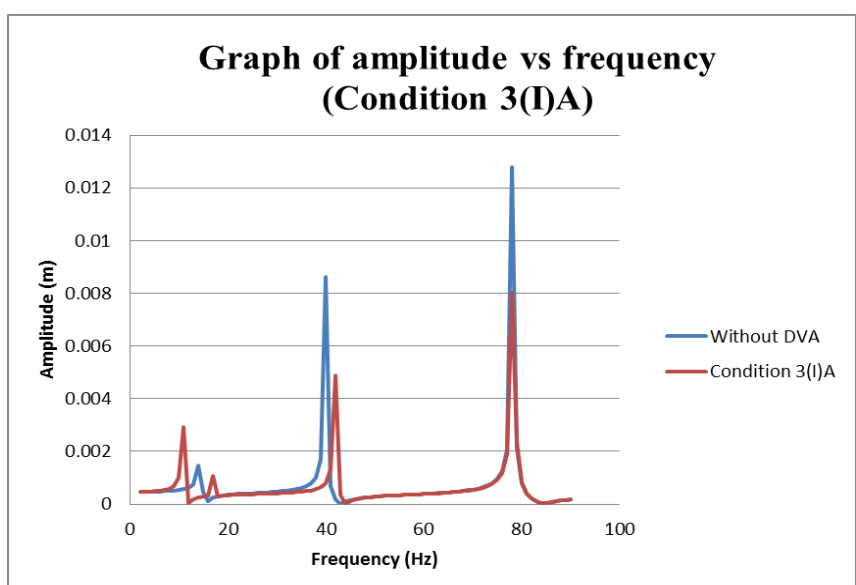

Figure-12: Graph of frequency response comparison between beam without DVA and Condition 3(I)A

\section{Result of the Condition 3(II)A}

Comparison between bare beam with this condition shows that the vibration amplitude of three modes managed to be reduced up to $92 \%$.

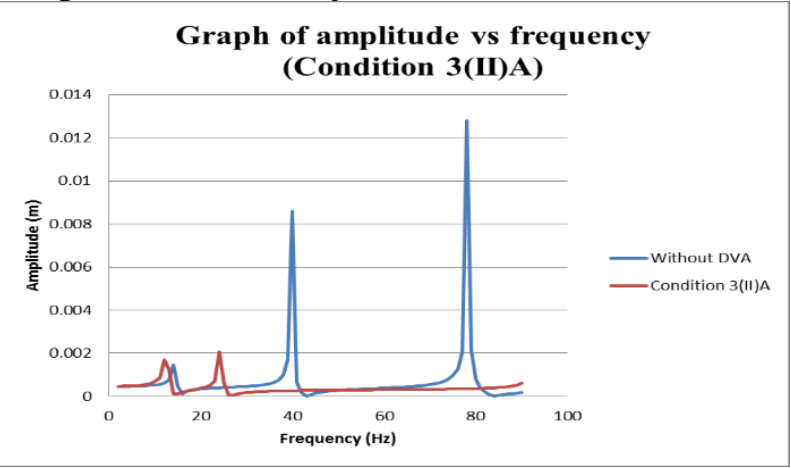

Figure-13: Graph of frequency response comparison between beam without DVA and Condition 3(II)A 


\section{Result of the Condition 3(III)A}

This condition display a result that almost similar with 'Condition 3(II)A'. However, the increase in stiffness of the spring has increase the amplitude of first vibration mode.

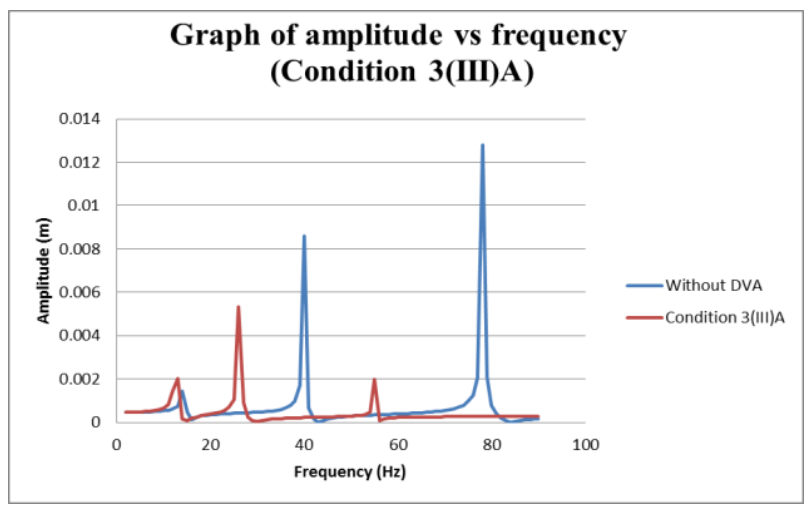

Figure-14: Graph of frequency response comparison between beam without DVA and Condition 3(III)A

Vibration amplitude reduction in first category of test
Results from Table- 3 shows that the placements of each DVA manage to absorb the vibration for every mode of vibration except when the DVA is located at node of third vibration mode. It leads to increment of vibration amplitude. The increment of amplitude occurred due to the inertia effect of third DVA at the vibration node. Although first mode is reduced to almost same value as third mode of vibration, it has lower percentage of reduction due to lower original amplitude value compare to third mode of vibration. Theoretically, each specific DVA is used to absorb vibration at particular mode but the DVA that design for specific vibration mode able to reduce the vibration for other mode of vibration. This is because the mass effect of the DVA has changed the original natural frequency of the beam. However, if the mass is placed at the node of specific vibration mode, the natural frequency will remain. Besides that, from the result, it is believed that the placement of DVA that near to both the vibrational antinode and excitation source is more effective. This finding can be observe by compare the result between placement of DVA in antinode of second vibration mode and antinode of third vibration mode. The best condition based on the Table- 3 is 'Condition 3(II)A where it contribute to most reduction of DVA in three modes of vibration. 
Table-3: Summary of percentage amplitude reduction for first category of test

\begin{tabular}{|c|c|c|c|c|c|c|}
\hline \multirow{2}{*}{ Mounting } & \multicolumn{2}{|c}{ First mode } & \multicolumn{2}{c|}{ Second mode } & \multicolumn{2}{c|}{ Third mode } \\
\cline { 2 - 7 } & Amplitude & Reduction & Amplitude & Reduction & Amplitude & Reduction \\
\hline Without DVA & $1.45 \times 10^{-3}$ & Ref. & $8.61 \times 10^{-3}$ & Ref. & $1.28 \times 10^{-2}$ & Ref. \\
\hline 1(I)A & $4.23 \times 10^{-4}$ & 70.74 & $7.99 \times 10^{-3}$ & 7.18 & $2.75 \times 10^{-3}$ & 78.55 \\
\hline 1(II)A & $3.98 \times 10^{-4}$ & 72.52 & $7.85 \times 10^{-3}$ & 8.85 & $3.57 \times 10^{-4}$ & 97.21 \\
\hline 1(III)A & $3.95 \times 10^{-4}$ & 72.72 & $7.88 \times 10^{-3}$ & 8.45 & $2.68 \times 10^{-4}$ & 97.91 \\
\hline 2(I)N & $4.23 \times 10^{-4}$ & 70.74 & $7.99 \times 10^{-3}$ & 7.18 & $2.75 \times 10^{-3}$ & 78.55 \\
\hline 2(II)N & $3.98 \times 10^{-4}$ & 72.52 & $7.85 \times 10^{-3}$ & 8.85 & $3.57 \times 10^{-4}$ & 97.21 \\
\hline 2(III)N & $3.95 \times 10^{-4}$ & 72.72 & $7.88 \times 10^{-3}$ & 8.45 & $2.68 \times 10^{-4}$ & 97.91 \\
\hline 2(I)A & $3.21 \times 10^{-4}$ & 77.84 & $5.67 \times 10^{-4}$ & 93.42 & $1.71 \times 10^{-3}$ & 86.68 \\
\hline 2(II)A & $2.18 \times 10^{-4}$ & 84.90 & $3.23 \times 10^{-4}$ & 96.25 & $4.25 \times 10^{-4}$ & 96.68 \\
\hline 2(III)A & $2.06 \times 10^{-4}$ & 85.764 & $2.99 \times 10^{-4}$ & 96.53 & $3.30 \times 10^{-4}$ & 97.43 \\
\hline 3(I)N & $3.78 \times 10^{-4}$ & 73.874 & $7.14 \times 10^{-4}$ & 91.70 & $1.77 \times 10^{-2}$ & -37.97 \\
\hline 3(II)N & $3.39 \times 10^{-4}$ & 76.584 & $3.91 \times 10^{-4}$ & 95.46 & $1.74 \times 10^{-2}$ & -35.62 \\
\hline 3(III)N & $3.34 \times 10^{-4}$ & 76.89 & $3.64 \times 10^{-4}$ & 95.78 & $1.78 \times 10^{-2}$ & -39.04 \\
\hline 3(I)A & $2.46 \times 10^{-4}$ & 83.02 & $7.97 \times 10^{-4}$ & 90.74 & $8.01 \times 10^{-3}$ & 37.42 \\
\hline 3(II)A & $1.11 \times 10^{-4}$ & 92.33 & $2.63 \times 10^{-4}$ & 96.95 & $3.52 \times 10^{-4}$ & 97.25 \\
\hline 3(III)A & $1.63 \times 10^{-4}$ & 88.73 & $2.20 \times 10^{-4}$ & 97.45 & $2.65 \times 10^{-4}$ & 97.93 \\
\hline
\end{tabular}

\section{Effect of DVAs placement at either node or antinode of the beam}

A set of simulation labeled according to alphabetical order are carried out to study the vibration characteristics of fixed ends beam after attaching multi DVA at specific location.

\section{Result for Condition A}

Two DVAs are attached at the antinode of beam in first and second vibration mode which first DVA attached at the middle of the beam and second DVA attached at $0.21 \mathrm{~m}$ from fixed end. All the three modes of vibration are reduced as shown in Figure-15. Although there are absent of third DVA in third mode of vibration, the vibration amplitude is still reduced. This is due to the mass of first DVA and second DVA that contribute for shifting of natural frequency in third vibration mode.

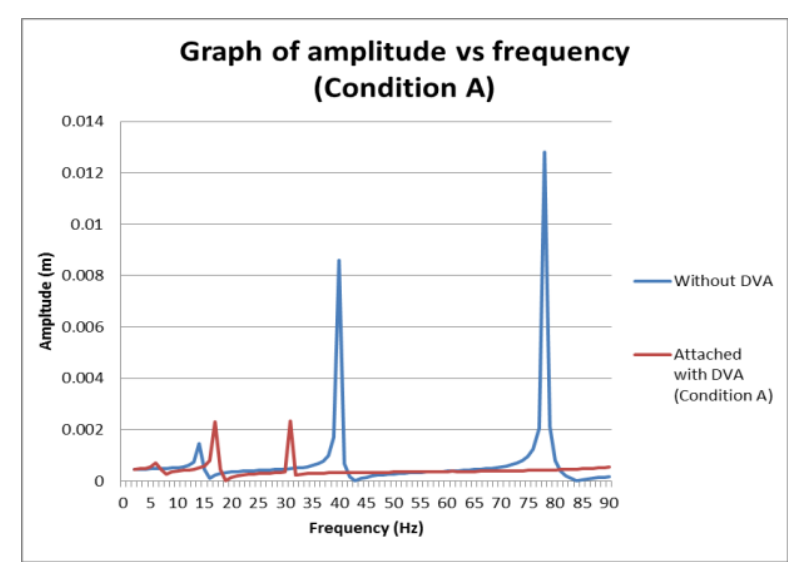

Figure-15: Graph of frequency response comparison between beam without DVA and Condition A

\section{Result for Condition B}

First DVA and third DVA are placed at its antinode of specific vibration for 'Condition B'. The location of antinode of these two modes is located at
$0.15 \mathrm{~m}$ and $0.42 \mathrm{~m}$ away from beam's fixed end. The DVA perform to absorb the vibration at first, second and third mode as shown in Figure-16. Although it contributes to large amplitude reduction at working frequency, the new natural frequency of the system is very near to natural frequency.

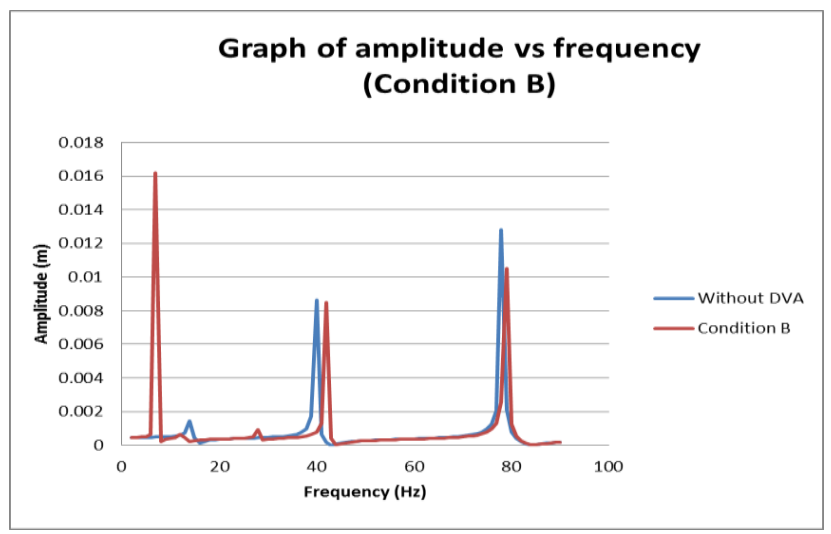

Figure-16: Graph of frequency response comparison between beam without DVA and Condition B

\section{Result for Condition C}

Two DVAs are attached separately at middle of the beam for second vibration mode and at $0.3 \mathrm{~m}$ from fixed end for third vibration mode. These two locations is the node for second and third mode of vibration. The reduction of vibration as shown in Figure-17 is due to the location for second DVA at the antinodes for first and third vibration mode while the location for third vibration near to antinode of second vibration. 


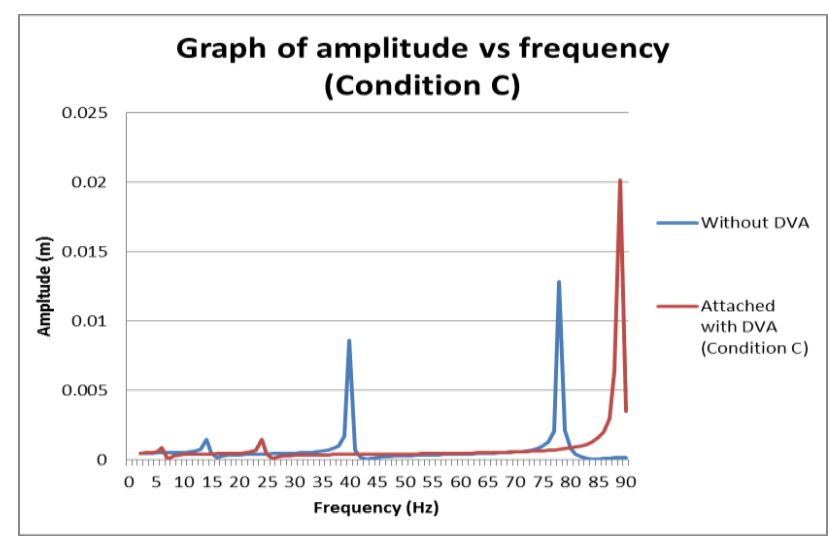

Figure-17: Graph of frequency response comparison between beam without DVA and Condition C

\section{Result for Condition D}

The simulation for this condition is almost same as 'Condition C' which it involve both second and third DVA. However, both the second DVA and third DVA are placed at antinode which located at $0.21 \mathrm{~m}$ and $0.42 \mathrm{~m}$ from beam's fixed end. Figure-18 shows the reduction in three modes of vibration. The reduction of first vibration mode is mainly due to the mass of third DVA that placed at the middle of the beam, which is also the location of antinode for first vibration mode.

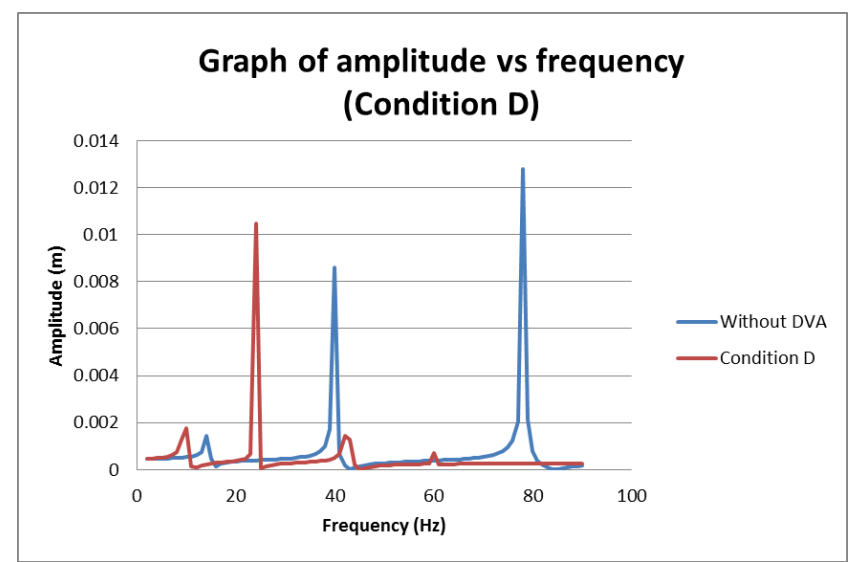

Figure-18: Graph of frequency response comparison between beam without DVA and Condition D

\section{Result for Condition $\mathbf{E}$}

First, second and third DVA are attached on the beam at location $0.42 \mathrm{~m}, 0.21 \mathrm{~m}$ and $0.15 \mathrm{~m}$ from fixed end respectively. These locations of DVAs are the antinode point of the respective vibration mode. Three modes of vibration are successfully reduced as shown in Figure-19. These reduction results occurred since the DVA for each mode are placed at the maximum antinode respectively

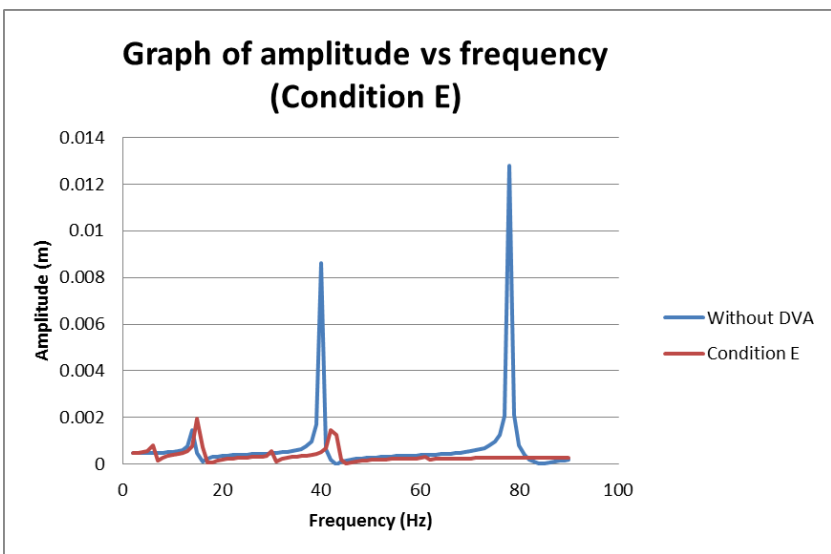

Figure-19: Graph of frequency response comparison between beam without DVA and Condition $\mathrm{E}$

\section{Comparison vibration amplitude reduction in second category of test}

The summary result of amplitude reduction is presented in Table-4. All the condition set able to absorb first vibration mode for at least $46 \%$. For second and third vibration mode, the amplitude of vibration reduced drastically. The ideal condition for vibration reduction occurs at Condition D where the amplitude reduction for overall mode of vibration is the highest. When 'Condition D' is attached with first DVA at first vibrational antinode, it consider as 'Condition E', the amplitude reduction for first mode of vibration decrease. The addition of DVA in such case didn't help in amplitude reduction of other vibration mode but increase the vibration of first vibration mode. Other than that, condition $\mathrm{A}, \mathrm{B}$ and $\mathrm{C}$ show that the DVA placed on the beam reduce the vibration of beam.

Table-4: Summary of percentage amplitude reduction for second category of test

\begin{tabular}{|c|c|c|c|c|c|c|}
\hline \multirow{2}{*}{ Mounting } & \multicolumn{2}{|c|}{ First mode } & \multicolumn{2}{c|}{ Second mode } & \multicolumn{2}{c|}{ Third mode } \\
\cline { 2 - 7 } & $\begin{array}{c}\text { Amplitude } \\
(\mathrm{m})\end{array}$ & $\begin{array}{c}\text { Reduction } \\
(\%)\end{array}$ & $\begin{array}{c}\text { Amplitude } \\
(\mathrm{m})\end{array}$ & $\begin{array}{c}\text { Reduction } \\
(\%)\end{array}$ & $\begin{array}{c}\text { Amplitude } \\
(\mathrm{m})\end{array}$ & $\begin{array}{c}\text { Reduction } \\
(\%)\end{array}$ \\
\hline $\begin{array}{c}\text { Without } \\
\text { DVA }\end{array}$ & $1.45 \times 10^{-3}$ & Ref. & $8.61 \times 10^{-3}$ & Ref. & $1.28 \times 10^{-2}$ & Ref. \\
\hline A & $5.13 \times 10^{-4}$ & 64.55 & $3.23 \times 10^{-4}$ & 96.25 & $4.25 \times 10^{-4}$ & 96.68 \\
\hline B & $2.11 \times 10^{-4}$ & 85.38 & $7.99 \times 10^{-4}$ & 90.72 & $2.56 \times 10^{-3}$ & 80.04 \\
\hline C & $3.98 \times 10^{-4}$ & 72.52 & $3.69 \times 10^{-4}$ & 95.71 & $7.37 \times 10^{-4}$ & 94.24 \\
\hline D & $2.30 \times 10^{-4}$ & 84.10 & $5.16 \times 10^{-4}$ & 94.01 & $2.65 \times 10^{-4}$ & 97.93 \\
\hline E & $7.78 \times 10^{-4}$ & 46.24 & $5.15 \times 10^{-4}$ & 94.02 & $2.65 \times 10^{-4}$ & 97.93 \\
\hline
\end{tabular}




\section{CONCLUSION}

In this study, an undamped dynamic vibration absorber has been attached on a fixed ends beam to reduce the vibration of first three modes of vibration. The attachment of DVAs occurs at vibration node or antinode of the vibration. Initially, the beam is operating at its own natural frequencies. The natural frequency of the fixed ends beam changed after attaching with DVAs. This is due to the effect of mass in DVA.

From the simulation results, it is prove that the DVA able to reduce the amplitude of vibration at working natural frequencies. The placement of DVA is a critical factor where it manages to obtain maximum vibration reduction when located at the antinode of the vibration depends on the mode of vibration. Additionally, when DVAs is located at node of vibration, it won't be able to absorb the vibration. The mass in DVA plays an important role in absorption of vibration because it will lower the natural frequency of the system. However, the natural frequency remains if the mass of DVA located at the node of vibration mode. When the mass of the beam increased, the natural frequency of the beam will decrease.

Besides that, it is prove that the number of DVA placed will not improve the reduction of vibration as long as the location of DVA is optimum. This result can be seen from comparison between condition 3(II)A and condition $\mathrm{E}$ where there is only one DVA involve in condition 3(II)A and three DVAs in condition E. Yet, the overall reduction of amplitude for condition 3(II)A is better. One DVA can be used to reduce the beam vibration for several modes for the reason that it is placed on the antinode or near the antinode of vibration mode.

\section{REFERENCES}

[1] Wang, C. Y., \& Wang, C. M. (2014). Structural Vibration: Exact Solutions for Strings, Membranes, Beams, and Plates. Florida: CRC Press.

[2] Cha, P. D., Dym, C. L., \& Wong, W. C. (1998). Identifying Nodes and Anti-Nodes of Complex Structures With Virtual Elements. Journal of Sound and Vibration, 211(1), 249-264.

[3] Kielb, R., Gavin, H., \& Dillenbeck, C. (2005). Tuned Vibration Absorbers: Analysis, Visualization, Experimentation, and Design. 1-10.

[4] Den Hartog, J. P. (1956). Mechanical vibration (4th ed.). New York: McGraw-Hill.

[5] Ormondroyd, J., \& Den Hartog, J. P. (1928). Theory of the dynamic vibration absorber. Transactions of the American Society of Mechanical Engineers.

[6] Zainulabidin, M. H., \& Jaini, N. (2012). Transverse Vibration of a Beam Structure Attached With Dynamic Vibration Absorbers: Experimental Analysis. International Journal of Engineering \& Technology IJET-IJENS, 12(06), 82-86.

[7] Aslamiah, M. J. (2016). Finite Element Analysis Of A Beam Structure Attached With Tuned Vibration Absorbers. Universiti Tun Hussein Onn Malaysia.

[8] Salleh, M. M., \& Zaman, I. (2016). Finite element modelling of fixed-fixed end plate attached with vibration absorber. ARPN Journal of Engineering and Applied Sciences, 11(4), 2336-2339.

[9] Akin, J. E. (2010). Finite Element Analysis Concepts Via SolidWorks (1st ed.). Texas: World Scientific. 